\title{
Case Report: An Occult Ipsilateral Femoral Neck Fracture Associated With Mid-Shaft Femur Fracture
}

\author{
Shahin Talebi ${ }^{1}$ D, Shayan Amjadi ${ }^{*}$ (D)
}

1. Orthopedic Research Center, Mazandaran University of Medical Science, Mazandaran, Iran.

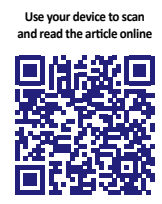

Citation Talebi Sh, Amjadi Sh. An Occult Ipsilateral Femoral Neck Fracture Associated With Mid-Shaft Femur Fracture. Journal of Research in Orthopedic Science. 2020; 7(4):185-188. http://dx.doi.org/10.32598/JROSJ.7.4.703.1

: http://dx.doi.org/10.32598/JROSJ.7.4.703.1

\section{(i) (5)}

Article info:

Received: 19 May 2020

Revised: 26 May 2020

Accepted: 01 Oct 2020

Available Online: 01 Nov 2020

Keywords:

Femoral fracture, Femoral neck fracture, Femoral shaft fracture

\begin{abstract}
A B S T RA C T
Occult ipsilateral femoral neck fractures associated with femoral shaft fractures are frequently missed. They may lead to adverse outcomes, such as fracture displacement and delayed treatment followed by poor outcomes, including delayed union, malunion, nonunion, and osteonecrosis, which may lead to early arthroplasty. These adverse effects can be prevented by the awareness of this combined injury pattern, the adequate preoperative and intraoperative imaging of the femoral neck, and proper treatment. A 27-year-old man was admitted with a displaced fracture of the right femur. Fluoroscopic evaluation was performed after the intramedullary nailing of the shaft fracture. The evaluation revealed an ipsilateral displaced femoral neck fracture, which was fixed with two cannulated screw inserted anterior to the nail. Between $2 \%$ and $9 \%$ of all femoral shaft fractures are associated with ipsilateral femoral shaft fractures. This case report necessitates the increase of awareness for the presence of associated femoral shaft and neck fractures in patients undergoing antegrade femoral nailing. Also, we recommend appropriate preoperative, intraoperative, and postoperative imaging.
\end{abstract}

\section{Introduction}

I

psilateral femoral neck fractures occur in up to $9 \%$ of femoral shaft fractures [1]; they typically result from a high-energy trauma in young patients [2]. However, more than one-third of ipsilateral femoral neck fractures are missed or discovered with a delay, after the treatment of femoral shaft fracture [2]. A failure to recognize an associated ipsilateral femoral neck fracture may result in fracture displacement, delayed treatment, and poorer outcomes, such as delayed union, malunion, nonunion, and osteonecrosis, which lead to early arthroplasty $[1,3]$.

These adverse effects can be prevented by the awareness of this combined injury pattern, adequate preoperative and intraoperative imaging of the femoral neck, and proper treatment [1]. During an operation, we diagnosed a femoral neck fracture through fluoroscopy, after the intramedullary nailing of the femoral shaft fracture; the fracture was then fixed.

\footnotetext{
* Corresponding Author:

Shayan Amjadi, MD.

Address: Orthopedic Research Center, Mazandaran University of Medical Science, Mazandaran, Iran.

Phone: +98 (912) 7018377

E-mail: shayanamajdi@yahoo.com
} 


\section{Case Presentation}

A 27-year-old man was admitted with a displaced fracture of the right femur (classified as AO/OTA type A1) (Figure 1). He had been involved in a motorcycle and car accident. The plain radiographs of the pelvic and knee showed no concomitant fracture (Figure 2). On clinical examination, we observed the apparent deformity and shortening of the lower limb and tendons at the fracture site. However, there was no tenderness in the hip. Moreover, it was not possible to examine the range of motion of the hip owing to the patient's pain at the fracture site. Thus, we performed the intramedullary locking nailing for the femur.

Intraoperative radiography was performed after the intramedullary nailing of the shaft fracture. The obtained radiographs revealed a vertical radiolucent line at the femoral neck, which was thought to be a further displacement of a hidden silent fracture or an iatrogenic fracture developed during nail insertion.

Because the fracture was minimally displaced, we decided to perform cancellous screw fixation. Also, technical difficulties were anticipated; the intramedullary rod was in the vicinity of the path of the screws. Furthermore, the removal of the intramedullary nail would risk displacing the femoral neck fracture. Finally, the patient was positioned on a traction table and the fracture was fixed with two cannulated screws inserted anterior to the nail (Figure 3).

\section{Discussion}

Between $2 \%$ and $9 \%$ of all femoral shaft fractures are associated with ipsilateral femoral shaft fractures $[4,5]$. The average age of patients is 35 years, also, $75 \%$ of patients
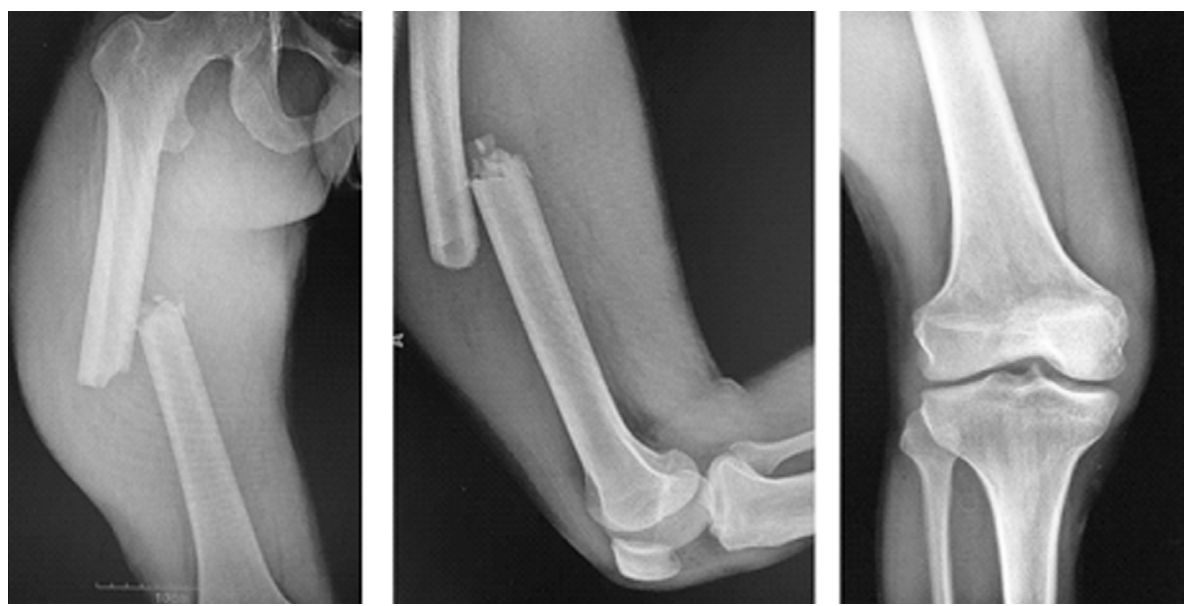

Figure 1. Plain X-rays of a 27-year-old man who sustained a high-energy femoral shaft fracture

Orthopedic Science

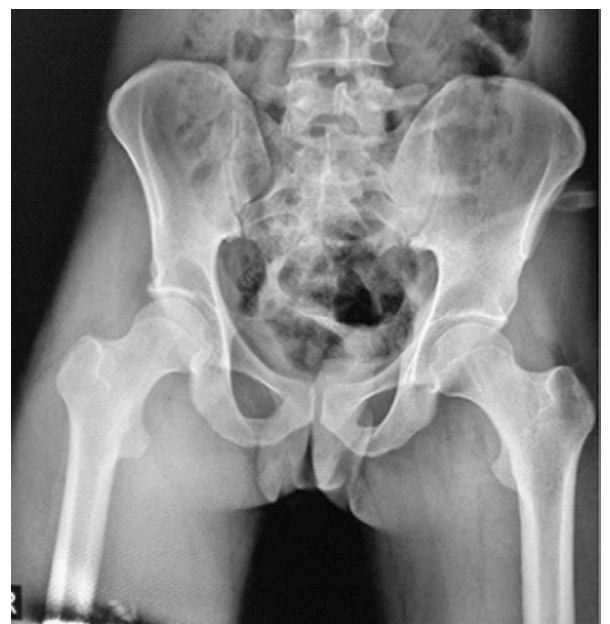

Figure 2. Pelvic X-ray with no fracture in ipsilateral femoral neck 

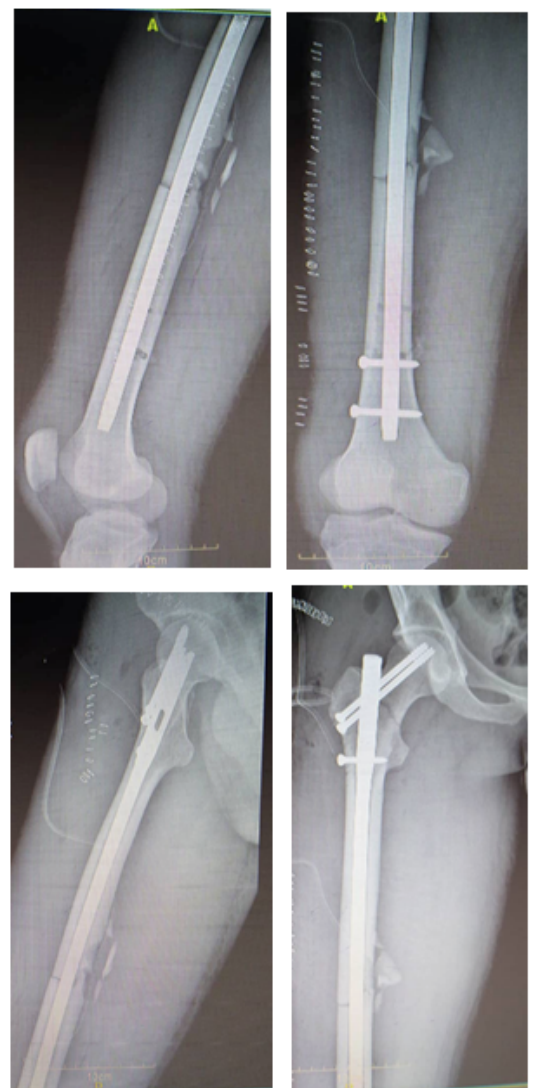

Figure 3. Anteroposterior and lateral views of postoperative x-ray of right femoral neck and shaft

Orthopedic Science

are male [4]. This uncommon pattern typically results from a high-energy trauma in young patients $[1,6,7]$.

The femoral shaft fracture is frequently comminuted in the middle third of the diaphysis because of the highenergy nature of this injury; this fracture is open in $15 \%$ to $33 \%$ of cases $[2,6]$. The femoral neck fracture is commonly basilar in location, vertical in orientation, and minimally displaced or nondisplaced in $60 \%$ of cases. Also, more than $75 \%$ of patients have multisystem injuries, and $20 \%$ to $40 \%$ of patients have ipsilateral knee injuries $[2,5]$. So, in this injury pattern, the knee and diaphyseal femur fractures absorb most of the energy, leading to a nondisplaced, easily missed femoral neck fracture [2]. Also, if the antegrade nailing of the starting site of femoral shaft fracture is placed too anteriorly, a stress riser can occur, leading to an iatrogenic femoral neck fracture [2].

Despite the increased awareness of the combined injury, they may still be missed in up to $11 \%$ of cases because of the following reasons. Many fractures are nondisplaced and difficult to visualize. Accurate diagnosis is limited by several technical factors, including poorquality radiographs, the lack of dedicated hip views, and the presence of overlying objects, such as traction splints that obscure the femoral neck, the presence of distracting injuries or other associated serious injuries in multiply injured patients, which direct the focus on more critical life-saving interventions $[2,8,9]$.

Once a high-energy diaphyseal femur fracture is diagnosed, it is necessary to perform dedicated hip imaging, including anteroposterior pelvis and lateral hip radiographs to evaluate the ipsilateral femoral neck [2]. If a CT (computed tomography) of the pelvis is ordered for other reasons, this is reviewed as a "free look" at the femoral neck $[1,10]$. Intraoperatively, the femoral neck should be evaluated under fluoroscopy, before, during, and after the fixation of a femoral shaft fracture [1, 11]. Besides, surgeons must be aware of the Mach band effect and pseudofractures and double-check the fracture line in the fluoroscopic image [12].

Also, it is recommended to obtain a good-quality plain radiograph view centered at the hip with $10^{\circ}$ to $15^{\circ}$ of hip internal rotation, following the fixation of any highenergy femoral shaft fracture. At follow-up, patients should be questioned about the presence of hip pain, and if positive, CT imaging is highly recommended. The preoperative diagnosis of femoral neck fractures can be 
more accurate with rapid limited-sequence Magnetic Resonance Imaging (MRI) [13].

Treatment options for ipsilateral femoral neck and shaft fractures include reconstruction nail, antegrade nail and separate screws adjacent to the nail, femoral neck screws and retrograde femoral nail, sliding hip screw with or without additional derotation screw and retrograde femoral nail, and femoral neck screws and the plate fixation of the shaft $[2,6,14,15]$. The screw fixation of the femoral neck would be extremely difficult in antegrade nailing; in such cases, computer navigation could enable the precise positioning of the guidewire and screws and minimize trauma to the bone [16].

\section{Ethical Considerations}

\section{Compliance with ethical guidelines}

This study was conducted based in the ethical guidelines of the Declaration of Helsinki, 1975, and the Patient provided an informed written consent for case information and associated images to be published.

\section{Funding}

This research did not receive any specific grant from funding agencies in the public, commercial, or not-profit sectors.

\section{Authors' contributions}

Conceptualization: Shahin Talebi; Investigation: Shayan Amjadi; Writing - original draft: Shayan Amjadi; Writing - review \& editing: Shahin Talebi, Shayan Amjadi; Supervision: Shahin Talebi.

\section{Conflict of interest}

The authors declared no conflict of interest.

\section{References}

[1] Jones CB, Walker JB. Diagnosis and management of ipsilateral femoral neck and shaft fractures. J Am Acad Orthop Surg. 2018; 26(21):e448-54. [DOI:10.5435/JAAOS-D-17-00497] [PMID]

[2] Hak DJ, Mauffrey C, Hake M, Hammerberg EM, Stahel PF. Ipsilateral femoral neck and shaft fractures: Current diagnostic and treatment strategies. Orthopedics. 2015; 38(4):247-51. [DOI:10.3928/01477447-20150402-05] [PMID]
[3] Heiney JP, Leeson MC, Vrabec GA. Delayed diagnosis of an ipsilateral femoral neck fracture with an associated femoral shaft fracture in light of a negative computed tomography scan. J Trauma. 2009; 67(4):E129-31. [DOI:10.1097/ TA.0b013e31805824ad] [PMID]

[4] Boulton CL, Pollak AN. Special topic: Ipsilateral femoral neck and shaft fractures-does evidence give us the answer? Injury. 2015; 46(3):478-83. [DOI:10.1016/j.injury.2014.11.021] [PMID]

[5] Wojcik K, Nowak R, Chmielewski L, Ochenduszka ST. Concomitant ipsilateral femoral neck and shaft fractures--analysis of cases. Ortop Traumatol Rehabil. 2009; 11(3):271-9.

[6] Ostrum RF, Tornetta P, Watson JT, Christiano A, Vafek E. Ipsilateral proximal femur and shaft fractures treated with hip screws and a reamed retrograde intramedullary nail. Clin Orthop Relat Res. 2014; 472(9):2751-8. [DOI:10.1007/s11999013-3271-5] [PMID] [PMCID]

[7] Peake C, Trompeter A. Low-energy atypical femoral shaft and ipsilateral neck fracture: A rare association. BMJ Case Rep. 2017; 2017:bcr2017222129. [DOI:10.1136/bcr-2017222129] [PMID] [PMCID]

[8] McDonald LS, Tepolt F, Leonardelli D, Hammerberg EM, Stahel PF. A cascade of preventable complications following a missed femoral neck fracture after antegrade femoral nailing. Patient Saf Surg. 2013; 7(1):16. [DOI:10.1186/1754-94937-16] [PMID] [PMCID]

[9] Deep K, Sharp I, Hay SM. Femoral neck fracture complicating intramedullary nailing of femoral shaft. Injury. 1999; 30(6):445-7. [DOI:10.1016/S0020-1383(99)00113-8] [PMID]

[10] Du CG, Zhang YZ, Chen W. [Progress in diagnosis and treatment of ipsilateral femoral neck and shaft fracture (Chinese)]. Zhonghua Wai Ke Za Zhi. 2016; 54(7):553-7. [DOI: 10.3760/cma.j.issn.0529-5815.2016.07.017] [PMID]

[11] Konig G, van Bergen P, Suren EG. [Femoral shaft fracture and secondary diagnosis of ipsilateral femoral neck fracture. Typical constellation or a complication of the Distal Femoral Nail (DFN)? (German)] Unfallchirurg. 2002; 105(7):656-9. [DOI: 10.1007/s00113-001-0409-9] [PMID]

[12] Yang KH, Kim S, Jeong JD. False femoral neck fracture detected during shaft nailing: A mach band effect. Yonsei Med J. 2013 54(3):803-5. [DOI:10.3349/ymj.2013.54.3.803] [PMID] [PMCID]

[13] Rogers NB, Hartline BE, Achor TS, Kumaravel M, Gary $\mathrm{JL}$, Choo AM, et al. Improving the diagnosis of ipsilateral femoral neck and shaft fractures: A new imaging protocol. J Bone Joint Surg Am. 2020; 102(4):309-14. [DOI:10.2106/ JBJS.19.00568] [PMID]

[14] Ostermann PA, Henry SL. Treatment of the ipsilateral femur shaft and femur neck fractures with the Russell-Taylor reconstruction nail. Chirurg. 1994; 65(11):1042-5. [PMID]

[15] Wruhs O, Jenny JY, Karger C. [Management of combination injuries of the femur shaft and femoral neck fractures with a locking nail. Multicenter study of 26 cases from 4 trauma clinics (German)]. Unfallchirurgie. 1986; 12(4):20814. [DOI:10.1007/BF02588460] [PMID]

[16] Chung FC, Wai CK, Koon WM. A case of screw fixation of femur neck fracture with the use of computer navigation in a woman with osteogenesis imperfecta. J Trauma. 2008; 65(5):1168-70. [DOI:10.1097/01.ta.0000197149.09525.9b] [PMID] 